\title{
SOURCES OF STARDUST IN THE GALAXY
}

\author{
ROBERT D. GEHRZ \\ Department of Astronomy, School of Physics and Astronomy, \\ 16 Church Street, S. E., University of Minnesota, \\ Minneapolis, $M N 55455$
}

\begin{abstract}
Observed mass loss rates and Galactic stellar population distributions are used to estimate the rate of injection of stardust into the ISM. M stars and RLOH/IR Stars produce most of the silicates; most of the carbon and SiC comes from carbon stars. WR stars, novae, and supernovae may eject dust with chemical anomalies. There is little observational evidence for a major stellar source of hydrocarbon grains. The Galactic dust ecology considered by comparing stellar dust injection with depletion by star formation and supernova shocks suggests that dust grains are produced by accretion in molecular clouds at 1 to 5 times the stellar rate.
\end{abstract}

\section{INTRODUCTION}

Dust in the interstellar medium (ISM) is produced in three principal ways: (1) by condensation in winds of evolved stars, (2) by condensation in ejecta from nova and supernovae, and (3) by accretion in dark clouds. I use observed stellar mass loss rates and Galactic population distributions to estimate the injection rate of stardust into the ISM. The mass loss rates are inferred by estimating the amount of material moving at escape velocity in outflows associated with all stellar populations that are currently observed in the Galaxy. We consider only the input to the Galactic disk.

\section{DISCUSSION}

A global assessment of the stardust input into the Galaxy requires determinations of (1) the Galactic distributions of mass-losing stars, (2) the average mass loss rates for these stars (3) the types of grains that condense in the outflows, and (4) the dust-to-gas mass ratio in the outflows.

\subsection{MODELING THE DUST INPUT FROM STELLAR POPULATIONS}

Evolved stellar systems exhibit both the steady-state, radiation driven, mass loss typical of giant/supergiant stars, and the explosive mass loss observed in novae and supernovae. In both cases, dust grains are often present in the ejecta. Gehrz and 
Woolf (1971) first suggested that infrared observations could be used to estimate the mass loss rates from stars with circumstellar dust. They developed an elementary radiation driven mass loss model from observations of $M$ stars and suggested that evolved stars could replenish the Galactic material consumed by star formation. Jura $(1984 a, 1984 b, 1986 b)$ has refined the model and applied it to many objects.

The dust mass returned to the ISM by a stellar disk population can be estimated for an exponential disk model (see Jones et al., 1981; and Bahcall, 1986) by evaluating the expression:

$$
\left(\frac{d M}{d T}\right)_{d u s t}=4 \pi \alpha^{2} \beta e^{R / \alpha} N_{i}\left\langle\frac{d M}{d t}\right\rangle F
$$

where $\alpha$ and $\beta$ are the $\mathrm{r}$ and $\mathrm{z}$ scale heights of the population, $R=8.7 \mathrm{kpc}$ is the distance of the sun from the Galactic center, $N_{i}$ is the number density of the population in the solar neighborhood, $\left\langle\frac{d M}{d t}\right\rangle$ is the mean mass loss rate for the stars in question, and $F$ is the ratio of dust to gas by mass. Values for $F$ are highly uncertain in many cases. Except as noted, we use $\alpha=2.2 \mathrm{kpc}$ (Jones et al., 1981; Bahcall, 1986) and the values for $\beta$ from Garwood and Jones (1987).

\subsection{THE DUST CONSTITUENTS IN STELLAR OUTFLOWS}

I summarize in Table 1 the current picture that infrared observations give about the chemical composition of the dust in stellar outflows. The primary mineral constituents in stellar ejecta are amorphous carbon, silicon carbide, and silicates (see Gehrz et al., 1987). Hydrocarbon molecules (PAH's, HAC's) may produce some of the infrared emission features observed in planetary nebulae, comets, and molecular cloud cores (see Allamandola et al., 1987). Although Hyland and MacGregor (1989) have reported possible hydrocarbon emission from a recent nova, and Gerbault and Goebel (1989) have argued that hydrocarbons may produce anomalous infrared emission from some carbon stars, there is currently no compelling evidence that hydrocarbon grains are an abundant constituent of the dust in stellar outflows. Generally, it appears that circumstellar hydrocarbon emission is seen only in sources with high excitation nebular conditions. There is circumstantial evidence that grains condensed in the ejecta of novae and supernovae may contain chemical anomalies similar to those in solar system meteorite inclusions (see Truran, 1985; Gehrz, 1988).

\subsection{GALACTIC DUST SOURCES}

I describe below the stellar populations believed to be injecting dust into the ISM and estimate their input in Table 2. Column 2 gives the total number of each stellar type in the Galaxy, column 3 estimates the gas to dust ratio in the outflow by mass, column 4 gives the total gas mass loss rate for the population, column 5 the total dust mass loss rate, and the notes beneath it comment upon the potential for various chemical enrichments. 


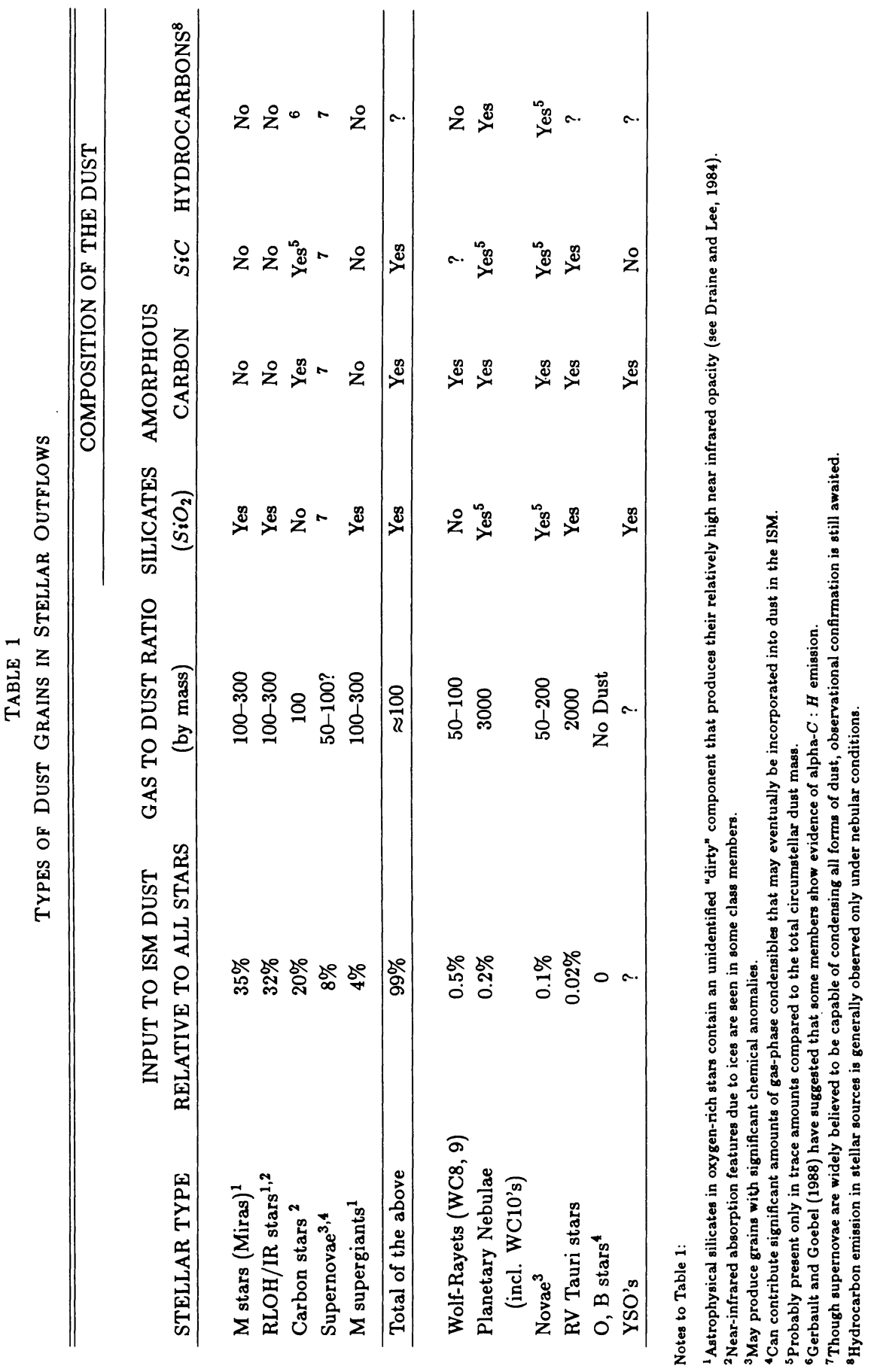


TABLE 2

Dust Production and Destruction on a Galactic Scale

\begin{tabular}{lcccc}
\hline \hline & TOTAL & GAS TO & $\begin{array}{c}\langle M / d t\rangle \\
\mathrm{M}_{\odot} \mathrm{yr}^{-1}\end{array}$ & $\begin{array}{c}(d M / d t)_{\text {dust }} \\
\mathrm{M}_{\odot} \mathrm{yr}^{-1}\end{array}$ \\
\hline M stars (Miras) & $9 \times 10^{6}$ & $100-300$ & $1.3-2.8$ & $0.004-0.028$ \\
RLOH/IR stars & $5.9 \times 10^{4}$ & $100-300$ & $1.0-3.0$ & $0.003-0.03$ \\
Carbon stars & $3-6 \times 10^{4}$ & $100-200$ & $0.34-0.96$ & $0.003-0.01$ \\
Supernovae & $1 /(30-50)$ yrs & $50-100$ & $0.1-0.3$ & $0.001-0.006$ \\
M supergiants & 5211 & $100-300$ & $0.05-0.5$ & $1.7-50 \times 10^{-4}$ \\
Wolf-Rayets & & & & \\
$\quad$ WN, WC7 & 2744 & - & 0.05 & none \\
$\quad$ WC8, WC9 & 484 & $50-100$ & 0.01 & $10^{-4}$ \\
Planetary Nebulae & $3.8 \times 10^{4}$ & 3000 & $0.05-0.5$ & $1.7-17 \times 10^{-5}$ \\
Novae & $30-50 \mathrm{yr}^{-1}$ & $50-200$ & $0.5-1.0 \times 10^{-4}$ & $10^{-5}-10^{-4}$ \\
RV Tauri stars & $600-1200$ & 2200 & $0.006-0.01$ & $3-5 \times 10^{-6}$ \\
O, B stars & & - & $0.03-0.3$ & none \\
\hline \hline
\end{tabular}

GALACTIC ECOLOGY

\begin{tabular}{llccc} 
Star formation & - & - & -3 to -10 & -0.03 to -0.1 \\
$\begin{array}{l}\text { Supernova shocks } \\
\text { Input from stars }\end{array}$ & - & - & - & -0.1 to -0.3 \\
\hline Total of the above & - & - & +2.9 to +8.4 & +0.01 to +0.08 \\
\hline $\begin{array}{l}\text { Dust Production in } \\
\text { Dark Clouds }\end{array}$ & - & - & 0 & -0.05 to -0.32 \\
\hline
\end{tabular}

Notes to Table 2:

${ }^{1}$ Possible sources of dust with ${ }^{22} \mathrm{Na},{ }^{26} \mathrm{Al}$, and $\mathrm{Xe}$ anomalies

${ }^{2}$ Possible sources of excess ${ }^{12} \mathrm{C},{ }^{14} \mathrm{~N}, 16,17 \mathrm{O},{ }^{22} \mathrm{Ne}$, and ${ }^{25,26} \mathrm{Mg}$ from nucleosynthesis

${ }^{3}$ Possible sources of dust with ${ }^{22} \mathrm{Na}$ and ${ }^{26} \mathrm{Al}$ anomalies

4 Sources of gas-phase condensibles

M STARS: The number densities given by Garwood and Jones (1987) and average mass loss rates from the data of Gehrz and Woolf (1971) and de Jager et al. (1988) were used for each spectral type from M0 to M9. Varying assumptions about scale heights and mass loss rates lead to a probable Galactic mass loss rate of 1.3 to 2.1 $\mathrm{M}_{\odot} \mathrm{yr}^{-1}$ for all $\mathrm{M}$ stars. If most of the dust is silicates and if all the silicon is condensed into grains, the gas to dust ratio in the outflow will be 250 to 300 (Gehrz and Woolf, 1971; Jura, 1984a). Jura (1987a) has pointed out that the ratio may be as high as 100/1. This may be a consequence of the fact that "dirty" rather than pure silicates are required to produce the large near infrared continuum dust opacity characteristic of the dust in oxygen-rich stars (see Draine and Lee, 1984). $\mathrm{M}$ stars therefore eject 0.004 to $0.020 \mathrm{M}_{\odot}$ of refractory silicates into the ISM per 
year.

RADIO LUMINOUS HYDROXYL MASER INFRARED (RLOH/IR) STARS: Herman and Habing (1985) estimate that the $\approx 60,000$ RLOH/IR stars in the Galaxy are each contributing $\approx 5.1 \times 10^{-5} \mathrm{M}_{\odot} \mathrm{yr}^{-1}$ for a total of $3 \mathrm{M}_{\odot} \mathrm{yr}^{-1}$ for the entire Galaxy.

CARBON STARS: We include in this classification all carbon rich objects including $\mathrm{C}, \mathrm{R}, \mathrm{N}$, and S stars as well as the enshrouded carbon stars discovered by the IRAS and AFGL surveys. Gehrz and Hackwell (1976) suggested, on the basis of a sample of objects from the AFGL sky survey, that obscured carbon stars might be a major source of mass input to the ISM. They argued that the high visual opacity of carbon caused carbon stars undergoing heavy mass loss to become an "invisible" population detectable only by deep infrared surveys. Jura $(1986 a, 1986 b)$ has confirmed this hypothesis using the IRAS survey and suggests that $C$ stars can account for as much as half of all the material in the ISM.

The average mass loss rates and total numbers for carbon stars are highly uncertain. The $S$ type stars number $\geq 10 \mathrm{kpc}^{-2}$ in the solar neighborhood and eject an average of $6 \times 10^{-8} \mathrm{M}_{\odot} \mathrm{yr}^{-1}$ (see Jura, 1988) so that their total Galactic contribution must be only $\approx 10^{-3} \mathrm{M}_{\odot} \mathrm{yr}^{-1}$. Claussen et al. (1987) found that the average mass loss rate for carbon stars detected by the CIT Two Micron Sky Survey (Neugebauer and Leighton, 1969) was only $2 \times 10^{-7} \mathrm{M}_{\odot} \mathrm{yr}^{-1}$, but noted that the Galactic population (some 30,000 stars) detected by this survey did not include the more heavily enshrouded carbon stars detected by the AFGL (see Price and Murdock, 1983) and IRAS surveys (IRAS, 1985) which appear to be losing mass at a much higher average rate of $2.3 \times 10^{-5} \mathrm{M}_{\odot} \mathrm{yr}^{-1}$ (see Jura, 1986a; and Knapp and Morris, 1985). Jura (1986a) has determined that these high mass loss stars are ejecting $1.4 \times 10^{-7} \mathrm{M}_{\odot} \mathrm{yr}^{-1}$ in carbon dust. The paucity of detailed knowledge about the spectral characteristics of the invisible carbon star populations detected by IRAS renders the Galactic distribution of such objects highly uncertain (see, for example, the very different points of view on this topic presented by Thronson et al., 1987, 1988; and Zuckermann, 1987). Thronson et al. (1987) suggest that the total number of carbon stars in the Galaxy is $28000[R / 15]^{2}$ where $R$ is the radius (kpc) of the Galaxy. For $R=15-25 \mathrm{kpc}, N=28,000-78,000$. Using an exponential disk model with $\beta=2 \mathrm{kpc}$ (Thronson et al., 1987) and a value of $\alpha=2.2 \mathrm{kpc}$ as for the $M$ stars, we would estimate from the local space density of carbon stars given by Thronson et al. (1987) that $N=63,000$. This latter number might be expected to include all extreme carbon stars. Thus we conclude that the mass return rate from carbon stars could be as high as $0.34-0.96 \mathrm{M}_{\odot} \mathrm{yr}^{-1}$. Given a gas to dust ratio of 100 by mass, the dust return rate is $0.03-0.01 \mathrm{M}_{\odot} \mathrm{yr}^{-1}$.

SUPERNOVAE: The condensation of all the common forms of astrophysical dust and of dust rich in chemical anomalies is expected to occur in supernovae on theoretical grounds (see Gehrz and Ney, 1987 and the references therein). There has been, to date, no observational verification. Given the observed supernova rate of 30-50 per year (see Truran, 1985; Jura, 1987a, 1987b), and presuming that the average supernova ejects $5-10 \mathrm{M}_{\odot}$ in the explosion, we project an input rate of $0.1-0.3$ $\mathrm{M}_{\odot} \mathrm{yr}^{-1}$. For normal gas-to-dust ratios, this might imply a dust mass loss rate as high as $0.001-0.007 \mathrm{M}_{\odot} \mathrm{yr}^{-1}$.

M SUPERGIANT STARS: Gehrz and Woolf (1971), Dupree (1986), and de Jager 
et al. (1988) find the mass loss rate for $M$ supergiants is $\approx 1-10 \times 10^{-5} \mathrm{M}_{\odot} \mathrm{yr}^{-1}$. There are about 5200 in the Galaxy (see Garwood and Jones, 1987), injecting a total of about $0.05-0.5 \mathrm{M}_{\odot} \mathrm{yr}^{-1}$ into the ISM. The dust in these sources is rich in silicates.

WOLF-RAYET STARS: Dust condensation has been observed in the WC8 star HD 193793 (Hackwell, Gehrz, and Grasdalen, 1979), an in addition, WC9 stars have thick carbon-rich dust shells (Gehrz and Hackwell, 1974). Although mass loss is common among all WR stars (Hackwell, Gehrz, and Smith, 1974), only the late WC stars appear to form stardust in the outflows. Abbott and Conti (1987) find that the average WR star wind conveys $2 \times 10^{-5} \mathrm{M}_{\odot} \mathrm{yr}^{-1}$ to the ISM, with $15 \%$ of the stars being WC8, 9 stars which have carbon dust in their ejecta. Based on the Galactic distribution of WR stars (Abbott and Conti, 1987; and Garmany, 1986), we find that the total mass input from WR stars is $\approx 0.01 \mathrm{M}_{\odot} \mathrm{yr}^{-1}$ with only $10^{-4} \mathrm{M}_{\odot}$ $\mathrm{yr}^{-1}$ being in the form of dust. Though this represents a negligible amount of dust compared to that input by other sources, there are significant chemical anomalies characteristic of the WR-star progenitor nucleosynthesis which could be contained in such dust grains. These anomalies include enrichments of ${ }^{12} \mathrm{C},{ }^{14} \mathrm{~N},{ }^{16,17} \mathrm{O},{ }^{22} \mathrm{Ne}$ and ${ }^{25,26} \mathrm{Mg}$ (Abbott and Conti, 1987). Thus, WR stars could be a source of some of the dust producing the anomalous inclusions seen in solar system meteorites (see Truran, 1985; and Gehrz, 1988). The early WR stars also put out large amounts of gas phase elements that may eventually be accreted onto ISM dust grains.

PLANETARY NEBULAE: There are 38,000 Planetary Nebulae in the Galaxy (Cahn and Wyatt, 1976) ejecting a total of 0.05-0.5 $\mathrm{M}_{\odot} \mathrm{yr}^{-1}$ (Natta and Panagia, 1981) into the ISM. Planetaries appear to condense silicates, carbon, and $S i C$ in their ejecta (Willner, 1984). The outflows are not rich in dust, with an average gas to dust ratio of 2000-3000 (Iyengar, 1986; Natta and Panagia, 1981); thus the dust returned to the ISM form these objects is $\approx 10^{-6} \mathrm{M}_{\odot} \mathrm{yr}^{-1}$. Pottasch et al. (1984) found that dust-to-gas ratio in the ejecta PN's decreases with increasing age which implies that PN's destroy dust as hard radiation eats its way out through the ejecta. Thus, PN's may be capable of destroying most of the dust formed in their ejecta before it reaches the ISM. However, the presence of an extended dust remnant from the PN phase of nova GK Per (see Bode et al., 1987) suggests that PN's do not destroy all the dust produced in the mass loss phase of the red giant precursor.

NOVAE: In almost all stellar populations, circumstellar dust formation and ejection is observed as a steady state process. The dynamical process of grain formation in a stellar wind was first observed in Galactic classical novae (see Ney and Hatfield, 1978; and Gehrz, 1988). The classical nova rate in the Galaxy is $\approx 30-50 \mathrm{yr}^{-1}$ (see Payne-Gaposchkin, 1957; Truran, 1985; and Downs, 1986), and the average mass ejection per eruption is $\approx 5-10 \times 10^{-5}$ (see Gallagher and Starrfield, 1978; Clayton, 1984; and Gehrz, 1988). The average mass input to the ISM from novae is therefore $0.0015-0.005 \mathrm{M}_{\odot} \mathrm{yr}^{-1}$. Since only $0.5-2 \%$ of the mass ejected in the average nova eruption condenses into dust, the total Galactic dust input from novae is only $\approx 10^{-5}$ to $10^{-1} \mathrm{M}_{\odot} \mathrm{yr}^{-1}$. However, novae may be a principal source of dust rich in such isotopes as ${ }^{22} \mathrm{Ne}$ and ${ }^{26} \mathrm{Mg}$ (see Truran, 1985; and Gehrz, 1988).

RV TAURI STARS: The 600-1200 Galactic RV Tauri stars lose mass at a rate as high as $10^{-5} \mathrm{M}_{\odot} \mathrm{yr}^{-1}$ (see Jura, 1986c) so that their total Galactic input is 0.006- 
$0.01 \mathrm{M}_{\odot} \mathrm{yr}^{-1}$. They may be sources of carbon, $S i C$, and silicates (see Gehrz, 1972). Because of their low metallicity, the gas-to-dust ratio of RV Tauri stars is probably as high as 2200/1 (Jura, 1986c), and they are not a major source of stardust.

OB STARS: Though 2.5-5 × $10^{4} \mathrm{O}$ and B stars are injecting $0.03-0.3 \mathrm{M}_{\odot} \mathrm{yr}^{-1}$ into the ISM (see Abbott, 1982; and Van Buren, 1985), the infrared energy distributions of these objects suggest that the outflows contain little dust.

OTHER SOURCES OF DUST: The energetic outflows of young stellar objects are known to contain dust (see Lada, 1985), but it is unclear whether grains are forming in the ejecta or whether they are remnants of the molecular cloud material from which the stars condensed. Seab (1987) has reviewed the evidence that refractory grains can be produced in dark clouds and the cores of GMC's. The rapid growth rates predicted for grains in clouds $\left(\approx 10^{6}-10^{7}\right.$ yrs.) implies that clouds could be efficient sources of ISM dust. Depletions in the ISM of heavy elements associated with dust (see Jenkins, 1987) are a strong indicator that any gas phase heavy elements ejected from stars are efficiently condensed onto ISM dust grains. Thus, WR stars, novae, and supernovae must be considered important contributors to ISM dust in so far as their ejecta can be highly metal rich.

\section{CONCLUSIONS: THE ECOLOGY OF STARDUST IN THE GALAXY}

We compare in Table 2 the rate of injection of stardust into the ISM with the rates of grain destruction by star formation and supernova shocks. Several conclusions can be drawn from the results summarized in Tables 1 and 2 .

1. $M$ stars, RLOH/IR stars, and $M$ supergiants are the primary sources of silicates while carbon stars, WR stars, and novae produce most of the carbon and $S i C$. Novae, supernovae, and WR stars may be responsible for most of the grains with chemical anomalies. There is currently no observational evidence for a substantial stellar source of hydrocarbon grains. If this is the case, then the production of hydrocarbon grains and hydrocarbon mantles on stardust may be primarily an ISM process.

2. The total estimated gas and dust inputs to the ISM for the sources in Table 2 are 2.9-8.4 and $0.01-0.08 \mathrm{M}_{\odot} \mathrm{yr}^{-1}$ respectively. An alternative estimate of the rate at which mass is returned to the ISM by evolving stars can be obtained from the white dwarf formation rate for stars with masses in the range from 0.8 to $10 \mathrm{M}_{\odot}$. The Present Day Mass Function (PDMF) of $N(M) \sim M^{-4}$ for such stars (see Miller and Scalo, 1979), combined with the nucleosynthesis time for main sequence stars $\left(t \sim M / L\right.$ where $\left.L \sim M^{3.3}\right)$ leads to a white dwarf formation rate of $7 \times 10^{-9} \mathrm{pc}^{-2} \mathrm{yr}^{-1}$ and a total mass input of about 9 $\mathrm{M}_{\odot} \mathrm{yr}^{-1}$. Assuming a gas-to-dust ratio of $50-100$ by mass, this yields a dust input of 0.09 to $0.18 \mathrm{M}_{\odot} \mathrm{yr}^{-1}$. These numbers compare favorably with the rates estimated in Table 2 . We conclude that the stellar populations listed in Table 2 represent a relatively complete accounting of the sources of mass input to the Galactic disk.

3. The current star formation rate inferred from the PDMF (Miller and Scalo, 1979 ) implies that star formation is depleting the ISM gas by some 3 to $10 \mathrm{M}_{\odot}$ $\mathrm{yr}^{-1}$ (see Miller and Scalo, 1979; Van Buren, 1985; Jura, 1987a). Assuming an 
ISM gas-to-dust ratio of 100 (Jura, 1987b), star formation depletes or destroys $0.03-0.1 \mathrm{M}_{\odot} \mathrm{yr}^{-1}$ of ISM dust. The rate of mass input to the ISM that we infer from the detailed accounting of mass loss by stellar populations (see Table 2) is roughly comparable. Jura $(\mathbf{1 9 8 7 b})$ has concluded that star formation is depleting the ISM at a rapid rate in the solar neighborhood, but the numbers in Table 2 suggest that this may not be true on a Galactic scale.

4. Seab (1987) has reviewed the hypotheses that ISM grains are rapidly destroyed by supernova shock waves on very short time scales $\left(1.7-5 \times 10^{8} \mathrm{yrs}\right.$.), and that grain formation in dark clouds must be important. He notes that the extreme depletion of refractory grain materials in the ISM is difficult to reconcile with the destruction hypothesis. Assuming the gas mass in the Galactic ISM is $5 \times 10^{9} \mathrm{M}_{\odot}$ with a gas-to-dust ratio of 100 (Seab, 1987), shocks will process 10-30 $\mathrm{M}_{\odot} \mathrm{yr}^{-1}$ in gas and destroy 0.1-0.3 $\mathrm{M}_{\odot} \mathrm{yr}^{-1}$ in dust. I estimate that $0.01-0.08 \mathrm{M}_{\odot} \mathrm{yr}^{-1}$ of dust is returned to the ISM by stars (see Table 2). This rate is inadequate to replenish the ISM dust. Given the range of uncertainties in the stardust production/grain destruction rates, it seems reasonable to conclude that a source of grains producing $\approx 1-5$ times as much dust as evolved stars is required to balance the deficit. Grain growth in dark clouds would appear to be the most attractive mechanism.

ACKNOWLEDGEMENTS. I thank T. A. Harrison, T. J. Jones, W. A. Stein, H. A. Thronson, and D. Whittet for useful discussions. I am supported by NASA, the United States Air Force, the National Science Foundation, and the University of Minnesota Graduate School.

\section{REFERENCES}

Abbott, D. C., and Conti, P. S. 1987, Ann. Rev. Astr. Ap., 25, 113.

Allamandola, L. J., Tielens, A. G. G. M., and Barker, J. R. 1987, in Interstellar Processes, eds. D. J. Hollenbach and H. A. Thronson, Jr., (Dordrecht: Reidel), p. 471.

Bahcall, J. N. 1986, Ann. Rev. Astr. Ap., $24,577$.

Bode, M. F., Seaquist, E. R., Frail, D. A., Roberts, J. A., Whittet, D. C. B., Evans, A., and Albinson, J. S. 1987, Nature, 829, 519.

Cahn, J. H., and Wyatt, S. P. 1976, Ap. J., 210, 508.

Claussen, M. J., Kleinmann, S. G., Joyce, R. R., and Jura, M. 1987, Ap. J. Suppl., $65,385$.

Clayton, D. D. 1984, Ap. J., 208, 144.

de Jager, C., Nieuwenhuijzen, H., and van der Hucht, K. A. 1988, Astr. Ap. Suppl. Ser., $72,259$.

Downes, R. A. 1986, Ap. J., 307, 170.

Draine, B. T., and Lee, H. M. 1984, Ap. J., 285, 89.

Dupree, A. K. 1986, Ann. Rev. Astr. Ap., $24,377$.

Garmany, C. D. 1986, in Luminous Stars and Associations in Galaxies, IAU Symposium No. 116., (Dordrecht: Reidel), p. 19.

Garwood, R., and Jones, T. J. 1987, Pub. A. S. P., 99, 453.

Gehræ, R. D. 1972, Ap. J., 178, 715.

. 1988, Ann. Rev. Astr. Ap., 25, in press.

Gehrz, R. D., Grasdalen, G. L., and Hackwell, J. A. 1987, in The Encyclopedia of Physical Science and Technology, $2,53$.

Gehr», R. D., and Hackwell, J. A. 1974, Ap. J., 194, 619.

. 1976, Ap. J. (Letters), 206, L161.

Gehrz, R. D., and Ney, E. P. 1987, Proc. Natl. Acad. Sci. USA, 84, 6961. 
Gehrz, R. D., and Woolf, N. J. 1971, Ap. J., 165, 285.

Gerbault, F., and Goebel, J. H. 1989, in Interstellar Dust: Contributed Papers, eds. A. G. G. M. Tielens and L. J. Allamandola, NASA CP-3036, in press.

Gallagher, J. S., and Starrfield, S. G. 1978, Ann. Rev. Astr. Ap., 16, 171.

Hackwell, J. A., Gehrz, R. D., and Grasdalen, G. L. 1979, Ap. J., $284,133$.

Hackwell, J. A., Gehrz, R. D., and Smith, J. R. 1974, Ap. J., $192,383$.

Herman, J., and Habing, H. J. 1985, in Reports on Progress in Physics, ESTEC.

Hyland, A. R., and McGregor, P. J. 1989, in Interstellar Dust: Contributed Papers, eds. A. G. G. M. Tielens and L. J. Allamandola, NASA CP-3036, in press.

IRAS Point Source Catalog. 1985, Joint IRAS Science Working Group (Washington, D. C.:USGPO). Iyengar, K. V. K. 1986, Astr. Ap., 158, 89.

Jenkins, E. B. 1987, in Interstellar Processes, eds. D. J. Hollenbach and H. A. Thronson, Jr., (Dordrecht: Reidel), p. 533.

Jones, T. J., Ashley, M., Hyland, A. R., and Ruelas-Mayorga, A. 1981, M. N. R. A. S., $197,413$.

Jones, T. W., Ney, E. P., and Stein, W. A. 1981, Ap. J., $250,324$.

Jura, M. $1984 a, A p$. J., 282, 200.

$1984 b, A p$. J., 286, 630.

$1986 a$, Ap. J., $\mathbf{8 0 8}, 327$.

1986 b, Irish Astr. J., 17, 322.

1986c, Ap. J., s09, 732 .

1987a, in Interstellar Processes, eds. D. J. Hollenbach and H. A. Thronson, Jr., (Dordrecht: Reidel), p. 3.

1987 b, 'Pub. A. S. P., 99, 1123.

- 1988, Ap. J. Suppl., 66, 33.

Knapp, G. R., and Morris, M. 1985, Ap. J., $292,640$.

Lada, C. J. 1985, Ann. Rev. Astr. Ap., 28, 267.

Miller, G. E., and Scalo, J. M. 1979, Ap. J. Suppl., 41, 513.

Natta, A., and Panagia, N. 1981, Ap. J., 248, 189.

Neugebauer, G., and Leighton, R. B. 1969, in Two Micron Sky Survey, (NAS SP-3047).

Ney, E. P., and Hatfield, B. F. 1978, Ap. J. (Letters), 219, L111.

Payne-Gaposchkin, C. 1957, in The Galactic Novae, (Amsterdam: North Holland Publ. Co.).

Pottasch, S. R., Baud, B., Beintema, D., Emerson, J., Habing, H. J., Harris, S., Houck, J., Jennings, R., and Marsden, P. 1984, Astr. Ap., 138, 10.

Price, S. D., and Murdock, T. L. 1983, in The Revised AFGL Infrared Sky Survey Catalog, (AFGLTR-83-0161).

Seab, C. G. 1987, in Interstellar Processes, eds. D. J. Hollenbach and H. A. Thronson, Jr., (Dordrecht: Reidel), p. 491.

Thronson, H. A., Jr., Latter, W. B., Black, J. H., Bally, J., and Hacking, P. 1987, Ap. J., 822 , 770.

Thronson, H. A., Jr., Latter, W. B., Black, J. H., Bally, J., and Hacking, P. 1988, Pub. A. S. P., submitted.

Truran, J. W. 1985, in Nucleosynthesis: Challenges and New Developments, p. 292.

Van Buren, D. 1985, Ap. J., 294, 567.

Willner, S. P. 1984, in Galactic and Extragalactic Infrared Spectroscopy, eds. M. F. Kessler and J. P. Phillips, (Dordrecht: Reidel), p. 37.

Zuckerman, B. 1987, in Cool Stars, Stellar Systems, and the Sun, eds. J. L. Linsky and R. E. Stencel, (Berlin: Springer Verlag), p. 351. 